\title{
QUADRATIC PARTITIONS: PAPER II
}

BY E. T. BELL

1. Equivalent Identities. ${ }^{*}$ We refer to I for the general notation, and now define a function $F$ of 4 arguments by

$$
F(w, z, u, v) \equiv F((z, u, v) \mid w)=-F(w, z,-v,-u) ;
$$

that is, $F$ is of the parity indicated and is arbitrary beyond the stated condition.

Obviously, $F(w, z, u, v)$ is an instance of the completely arbitrary parity function $f((z, u, v) \mid w)$ having parity $p(3 \mid 1)$, and $f((z, u, v) \mid w)-f((z,-v,-u) \mid w)$ is an instance of $F(w, z, u, v)$. Let $\sum_{i}$ refer to a set of values of $(w, z, u, v)$. Then, by what precedes,

$$
\begin{gathered}
\sum_{i} c_{i} F\left(w_{i}, z_{i}, u_{i}, v_{i}\right)=0 \\
\sum_{i} c_{i}\left[f\left(\left(z_{i}, u_{i}, v_{i}\right) \mid w_{i}\right)-f\left(\left(z_{i},-v_{i},-u_{i}\right) \mid w_{i}\right)\right]=0
\end{gathered}
$$

are both true or both false. If both are true, and hence if either is true, the relations are called equivalent. Many instances of equivalent identities will occur in these notes. The equivalence is always, as above, immediate from the properties of the functions implied in the parity notation, and need not be further discussed.

2.,$\phi$ Identities. Combined with the transformation of the second order, Jacobi's formula for the product of $4 \vartheta$-functions gives an endless chain of identities between functions $\vartheta, \phi$, which can be written down by elementary algebra. $\uparrow$ These lead to identities between parity functions in any number of arguments summed over quadratic partitions. The first of these $\vartheta, \phi$ identities to be discussed is

* The preceding note, this Bulletin, vol. 37 (1931), pp. 870-5, will be cited as I.

$\dagger$ At the writer's request, Mr. W. H. Gage of Victoria College, B.C., has derived a large number of these identities. A particular identity can easily be checked independently; Mr. Gage's systematic derivations reveal the simple interconnections between all. A sufficient account of the method will doubtless appear elsewhere. 


$$
\begin{aligned}
\Phi(w, z, u, v)+\Phi(w, & -z,-u,-v) \\
& -\Phi(w,-z, v, u)-\Phi(w, z,-v,-u) \equiv 0,
\end{aligned}
$$

where $\Phi(w, z, u, v)$ denotes

$$
\phi_{001}\left(\frac{w+z}{2}, u\right) \vartheta_{3}\left(\frac{v-z}{2}, q^{1 / 2}\right) \vartheta_{3}\left(\frac{w-u}{2}, q^{1 / 2}\right) .
$$

The required expansions in connection with this are

$$
\begin{aligned}
\vartheta_{3}\left(x, q^{1 / 2}\right) & =\sum q^{\nu^{2}} \cos 2 \nu x, \\
\phi_{001}(x, y) & =\csc y+4 \sum q^{n}\left[\sum \sin (2 t x+\tau y)\right] .
\end{aligned}
$$

3. Passage to Parity Functions. The above $\vartheta, \phi$ identity implies and is implied by that obtained by equating to zero the coefficient of $q^{n}$, namely,

$$
\begin{aligned}
& 4 \sum \sin \left(t+\nu_{2}\right) w\left[\cos \left\{\left(t-\nu_{1}\right) z+\left(\tau-\nu_{2}\right) u+\nu_{1} v\right\}\right. \\
& \left.\quad-\cos \left\{\left(t-\nu_{1}\right) z-\nu_{1} u-\left(\tau-\nu_{2}\right) v\right\}\right] \\
& +\epsilon_{2}(n) \sum \sin b w[\csc u \sin (a z+b u-a v) \\
& \quad-\csc v \sin (a z+a u-b v)]=0,
\end{aligned}
$$

the summations referring to the partitions $n=2 t \tau+\nu_{1}{ }^{2}+\nu_{2}^{2}$, $n=a^{2}+b^{2}$, where $\epsilon_{2}(n)=1$ or 0 according as $n$ is or is not a sum of 2 squares. Refer to the first identity in I, $\$ 7$. Then we pass at once to

$$
\begin{gathered}
4 \sum F\left(t+\nu_{2}, t-\nu_{1}, \tau-\nu_{2}, \nu_{1}\right) \\
=\epsilon_{2}(n) \sum \operatorname{sgn} b\left[\{1-e(b)\} F(b, a, a, 0)+2 \sum_{r=1}^{B} F(b, a, a, 2 r-e(b))\right],
\end{gathered}
$$

with $F$ as in $\$ 1$, and $B \equiv\left[\frac{1}{2}|b|\right]$.

4. Form for $F$ Entire in $(u, v)$. Denote by $F_{1}(w, z, u, v)$ the function obtained from $F(w, z, u, v)$ by restricting the latter to be entire in $(u, v)$, and apply to the trigonometric identity in $\S 3$ the first formula in the last pair of $I, \S 6$. Then

$$
4 \sum F_{1}\left(t+\nu_{2}, t-\nu_{1}, \tau-\nu_{2}, \nu_{1}\right)=\epsilon_{2}(n) \sum F_{1}\left(b, a, a, \rho^{\prime}(b)\right) .
$$

In the next note we shall indicate how this may be written down at once from $\$ 3$, end. 
5. Contraction. We describe the general process for $f\left(\xi_{1}, \cdots\right.$, $\left.\xi_{r} \mid \eta_{1}, \cdots, \eta_{s}\right)$ as in I, $\$ 2$. This function has the same parity as

$$
f_{1}\left(\xi_{p}, \cdots, \xi_{t} \mid\right) f\left(\xi_{1}, \cdots, \xi_{r} \mid \eta_{1}, \cdots, \eta_{s}\right) \text {, }
$$

where $p, \cdots, t$ are distinct integers of the set $1, \cdots, r$, and $f_{1}$ is arbitrary beyond the parity implied in the notation. The order of a vector is tlie number of its coordinates. Let $P, \cdots, T$ be the respective orders of $\xi_{p}, \cdots, \xi_{t}$. Let $\{N\}$ denote for the moment the zero vector of order $N$. Choose for $f_{1}$ the function defined by

$$
\begin{gathered}
f_{1}(\{P\}, \cdots,\{T\} \mid)=1 \\
f_{1}\left(\xi_{p}, \cdots, \xi_{t} \mid\right)=0,\left(\xi_{p}, \cdots, \xi_{t}\right) \neq(\{P\}, \cdots,\{T\}) .
\end{gathered}
$$

Let $\xi_{i}{ }^{h}, \eta_{j}{ }^{h}$, where $h$ denotes a superscript, be a set of values of $\xi_{i}, \eta_{j}$. Then, from a relation of the type

$$
\sum_{h} c_{h} f\left(\xi_{1}{ }^{h}, \cdots, \xi_{r}{ }^{h} \mid \eta_{1}{ }^{h}, \cdots, \eta_{s}{ }^{h}\right)=0,
$$

we infer the like with $\xi_{p}{ }^{h}, \cdots, \xi_{t}{ }^{h}$ suppressed. For, the product $f_{1} f$ is an instance of $f$.

Let $\xi_{i}{ }^{h}, \eta_{j}{ }^{h}$ refer to a partition, say $\pi$. Then, from $\pi$ and $\xi_{p}{ }^{h}=\{P\}, \cdots, \xi_{t}{ }^{h}=\{T\}$, at least one of the indeterminates can be eliminated. There results a new partition in fewer indeterminates, restricted by all the conditions which express the arithmetical character (sign, integrality, evenness or oddness, etc.) of the eliminated indeterminates. The $\sum_{h}$ with $\xi_{p}{ }^{h}, \cdots, \xi_{t}{ }^{h}$ suppressed thus refers to the new or contracted partition, and we say that $f\left(\xi_{1}, \cdots, \xi_{r} \mid \eta_{1}, \cdots, \eta_{s}\right)$ has been contracted with respect to $\xi_{p}, \cdots, \xi_{t}$.

6. Application of $\S 5$ to $\S \S 3,4$. We contract $F(w, z, u, v)$ with respect to $z^{*}$. The partitions in $\$ 3$ contract to

$$
n=x y+z^{2}, n=t^{2},
$$

the first of which is restricted,

$$
x>0, y-x>0, y-x \equiv 2 \bmod 4, z \gtreqless 0,
$$

and $t$ in the second, by the general notation in I, $\S 3$, is $>0$. The function $F(w, z, u, v)$ in $\S 3$ is replaced by

$$
G(w, u, v) \equiv G((u, v) \mid w)=-G((-v,-u) \mid w),
$$

* There can be no confusion between the argument $z$ in $F$ and $z$ in the partition, as the partition is defined by the conditions stated. 
and we have

$$
\sum G\left(x+z, \frac{y-x}{2}-z, x\right)=\epsilon_{1}(n) \sum_{r=1}^{T} G(t, 0,2 r-e(t)),
$$

where $\epsilon_{1}(n)=1$ or 0 according as $n$ is or is not a square $>0$, and $T=[t / 2]$.

Similarly, from $\$ 4$, if $G_{1}(w, u, v)$ is $G(w, u, v)$ with the restriction of entirety in $(u, v)$ we get

$$
\begin{aligned}
4 \sum G_{1}\left(x+z, \frac{y-x}{2}\right. & -z, x) \\
& =\epsilon_{1}(n)\left[G_{1}\left(t, 0, \rho^{\prime}(t)\right)-G_{1}\left(t, 0, \rho^{\prime}(-t)\right)\right] .
\end{aligned}
$$

California Institute of Technology

THE TRANSFORMATION OF LINES OF SPACE BY MEANS OF TWO QUADRATIC REGULI*

BY A. R. WILLIAMS

If we take two quadratic reguli, a line $l$ meets two generators of each. To $l$ we make correspond the other transversal of the four generators. This involutory transformation of the lines of space is one of three, quite similar in principle. $\dagger$ This case admits a very simple and effective algebraic treatment without the use of hyperspace.

We may take for the equations of two non-singular quadrics with real rulings $x_{1}^{2}+x_{2}^{2}-x_{3}^{2}-x_{4}^{2}=0$ and $a^{2} x_{1}^{2}+b^{2} x_{2}^{2}-c^{2} x_{3}^{2}$ $-d^{2} x_{4}^{2}=0$. On the former lies the regulus $R_{1}$ defined by $x_{1}-x_{3}=m\left(x_{4}-x_{2}\right), x_{1}+x_{3}=1 /\left(m\left(x_{4}+x_{2}\right)\right)$. The Plücker coordinates of a line of this regulus are

(1) $p_{12}: p_{13}: p_{14}: p_{23}: p_{42}: p_{34}$

$$
=\left(m^{2}+1\right): 2 m:\left(m^{2}-1\right):\left(m^{2}-1\right): 2 m:-\left(m^{2}+1\right) .
$$

The other regulus $R_{1}^{\prime}$ on the same quadric is given by the

* Presented to the Society, November 28, 1932.

$\dagger$ Discussed by Mr. J. M. Clarkson in the present issue of this Bulletin, vol. 38 , pp. 533-540. 